\title{
UNORIENTED BRANCHED COVERINGS ARISING FROM GROUP ACTIONS
}

\author{
VIRGINIA R. YOUNG
}

\begin{abstract}
For an unbranched covering $f: M^{n} \rightarrow N^{n},[M]=(\operatorname{deg} f)[N]$ in unoriented cobordism $\Re_{*}$. Thus, in general, if $f: M \rightarrow N$ is a branched covering, then $[M]-(\operatorname{deg} f)[N]$ depends upon the branching behavior.

In this note we describe the ideal $I_{G}$ of unoriented cobordism classes $\left[M^{n}\right]-$ $|G|\left[M^{n} / G\right]$, where $G$ is a finite group acting on $M$ so that $M \rightarrow M / G$ is a $|G|$-fold smooth branched covering of closed smooth manifolds.
\end{abstract}

1. Introduction. The purpose of this note is to describe the ideal $I_{G}$ of unoriented cobordism classes [ $\left.M^{n}\right]-|G|\left[M^{n} / G\right]$, where $G$ is a finite group acting on $M$ so that $M \rightarrow M / G$ is a $|G|$-fold smooth branched covering of closed smooth manifolds.

It is known that for an unbranched covering $f: M^{n} \rightarrow N^{n},[M]=(\operatorname{deg} f)[N]$ in unoriented cobordism $\mathfrak{N}_{*}$. Thus, in general, if $f: M \rightarrow N$ is a branched covering, then $[M]-(\operatorname{deg} f)[N]$ depends upon the branching behavior.

We follow Brand [1] in defining branched covering, including a smoothness condition:

Definition. A branched covering is a smooth map $f: M^{n} \rightarrow N^{n}$ between closed smooth manifolds which is finite-to-one and open. The singular set is the set of points of $M$ at which $F$ is not a local homeomorphism, and the branch set $B_{f}$ is the image under $f$ of the singular set. We assume that $B_{f}$ is a smoothly embedded codimension 2 submanifold of $N$.

Let $B_{k}^{n-2}$ be a component of $f^{-1} B_{f}$. Then, on a neighborhood of $B_{k}, f$ is locally isomorphic to the map $D^{n-2} \times D^{2} \rightarrow D^{n-2} \times D^{2}:(x, z) \rightarrow\left(x, z^{k}\right)$. The number $k$ is called the local branching degree. For more details, see Stong [5].

Note that $M-f^{-1} B_{f} \rightarrow N-B_{f}$ is an unbranched covering. If $M$ and $N$ are path-connected and if $\pi_{1}\left(N-B_{f}\right) / \pi_{1}\left(M-f^{-1} B_{f}\right) \simeq G$, a finite group with $|G|=$ $\operatorname{deg} f$, then $G$ acts on $M$, freely on $M-f^{-1} B_{f}$, with cyclic isotropy groups, and $M \rightarrow M / G=N$ is the branched covering.

Conversely, if $G$ acts on $M$ with cyclic isotropy groups such that $\{x \in M$ : $\left.G_{x} \neq\{1\}\right\}$ is a codimension 2 submanifold of $M$, then $M \rightarrow M / G$ is a branched covering. If $F_{k}$ is a component of the fixed point set of $\mathbf{Z}_{k} \subset G$, then $k$ is the local branching degree on $F_{k}$.

Received by the editors February 27, 1984.

1980 Mathematics Subject Classification. Primary 57S17; Secondary 57R75.

(1) 1985 American Mathematical Society $0002-9939 / 85 \$ 1.00+\$ .25$ per page 
Notation. If $G=\mathbf{Z}_{d}$, then we write $I_{d}$ for $I_{G}$. We write $\mathbf{R} P\left(n_{1}, \ldots, n_{k}\right)$ to mean the real projective space of the bundle $\gamma_{n_{1}} \oplus \cdots \oplus \gamma_{n_{k}}$ sitting over $\mathbf{R} P^{n_{1}} \times \cdots \times$ $\mathbf{R} P^{n_{k}}$, where $\gamma_{n_{i}}$ is the standard line bundle over $\mathbf{R} P^{n_{i}}[\mathbf{6}]$.

In $\S 2$, we determine $I_{G}$ in unoriented cobordism $\mathfrak{N}_{*}$ for all finite groups $G$. We begin by noting the following reduction of the problem:

Proposition A. If $G$ is a finite group with a Sylow 2-subgroup $S$, then $I_{G}=I_{S}$ in $\mathfrak{Y}_{*}$.

Stong [6] shows that $I_{2}=\oplus_{n}\left\{\alpha \in \mathfrak{N}_{n}: w_{1}^{n}(\alpha)=0\right\}$ in $\mathfrak{l}_{*}$.

Proposition B. For $s>1, I_{2^{\prime}}=\oplus_{n}\left\{\alpha \in \mathfrak{N}_{n}: w_{1}^{i} w_{n-i}(\alpha)=0,1 \leqslant i \leqslant n\right\}$ in $\mathfrak{i}_{*}$.

Proposition C. If $S$ is a 2-group containing $\mathbf{Z}_{2} \times \mathbf{Z}_{2}$, then $I_{S}=0$ in $\mathfrak{R}_{*}$.

If $S$ is a nonabelian 2-group that does not contain $\mathbf{Z}_{2} \times \mathbf{Z}_{2}$, then $S$ is a generalized quaternion group. Therefore, we finish our study with the following:

'Proposition D. If $Q$ is a generalized quaternion group, then $I_{Q}$ in $\mathfrak{N}_{*}$ is the ideal generated by $[\mathbf{R} P(0, m, 2 j+1,2 j+1)], m \geqslant 4 j+3$.

I thank my advisor, Robert E. Stong, for suggesting this problem and for his continual support and encouragement.

2. Unoriented branched coverings. Many of our results rely upon the following proposition due to Stong [5].

Proposition 2.1. If $f: M^{n} \rightarrow N^{n}$ is a branched covering, the $[M]-(\operatorname{deg} f)[N]=$ $\left[\mathbf{R} P\left(\nu_{\text {even }} \oplus 1\right)\right]$ in $\mathfrak{N}_{*}$, where $\nu_{\text {even }}=\bigcup_{k \text { even }} \nu_{k}$, with $\nu_{k}$ the normal bundle of $B_{k}$ in $M$.

The following reduction of the problem follows from the above result.

Proposition 2.2. If $G$ is a finite group with a Sylow 2-subgroup $S$, then $I_{G}=I_{S}$ in $\mathfrak{H}$ *

Proof. If $M^{n} \rightarrow M^{n} / G$ is a branched covering, then

$$
\begin{aligned}
{[M]-|G|[M / G] } & =[M]-|S|[M / S]+|S|\{[M / S]-[G: S][M / G]\} \\
& =[M]-|S|[M / S],
\end{aligned}
$$

and $M \rightarrow M / S$ is a branched covering. Thus, $I_{G} \subset I_{S}$. (Note: By Proposition 2.1, if $S=\{1\}$, then $[M]-|G|[M / G]$ is 0 because $M \rightarrow M / G$ is a branched covering with only odd local branching degrees.)

If $M \rightarrow M / S$ is a branched covering, then $G$ acts on $G \times{ }_{S} M$ as multiplication on $G$. $G \times{ }_{S} M \rightarrow G \times{ }_{S} M / G \simeq M / S$ is a branched covering with [ $\left.G \times{ }_{S} M\right]-$ $|G|[M / S]=[G: S][M]-|G|[M / S]=[G: S]\{[M]-|S|[M / S]\}=[M]-|S|[M / S]$. Thus $I_{S} \subset I_{G}$. $\quad \square$

ObSERVATION. According to Stong [6], $I_{2}=\left\{\alpha: w_{1}^{n}(\alpha)=0\right\}$ in $\Re_{*}$.

Proposition 2.3. For $s>1, I_{2^{\prime}}=\left\{\alpha: w_{1}^{i} w_{n-i}(\alpha)=0,0 \leqslant i \leqslant n\right\}$ in $\mathfrak{l i}_{*}$. 
Proof. Let $M^{n} \rightarrow M^{n} / \mathbf{Z}_{2^{\text {s }}}$ be a branched covering, and let $F^{n-2} \subset M^{n}$ be the singular set. $F=A \cup B$, where $A$ is the set of points with isotropy group $\mathbf{Z}_{2}$ and $B=F-A$.

Let $\nu \rightarrow F$ be the normal bundle of $F$ in $M$, and let $c \in H^{1}\left(\mathbf{R} P(\nu+1) ; \mathbf{Z}_{2}\right)$ be the first Stiefel-Whitney class of the double cover by the sphere bundle. We will often consider $c$ to be the corresponding class in $H^{1}\left(\mathbf{R} P(\nu) ; \mathbf{Z}_{2}\right)$ because the classifying map $\mathbf{R} P(\nu) \rightarrow \mathbf{R} P^{\infty}$ factors through the inclusion and classifying map $\mathbf{R} P(\nu) \rightarrow$ $\mathbf{R} P(\nu+1) \rightarrow \mathbf{R} P^{\infty}$. If $w(\nu)=1+u_{1}+u_{2}$, then $w(\mathbf{R} P(\nu))=w(F)(1+c)^{2}+$ $u_{1}(1+c)+u_{2}=w(F)\left(1+u_{1}\right)$ since $c^{2}+c u_{1}+u_{2}=0$. Because $\left.\nu\right|_{B} \rightarrow B$ is a complex vector bundle, $\left.u_{1}\right|_{B}=0$, implying that $w(\mathbf{R} P(\nu))=w(A)\left(1+u_{1}\right)+w(B)$.

$\mathbf{Z}_{2^{*}} / \mathbf{Z}_{2} \simeq \mathbf{Z}_{2^{s-1}} \neq\{1\}$ acts freely on $A . \mathbf{Z}_{2}$ acts as multiplication by -1 in the fibers of $\left.\nu\right|_{A} \rightarrow A$; thus, $\mathbf{Z}_{2}$ acts as $\operatorname{det}\left(-I_{2}\right)=1$ on $\left.\operatorname{det} \nu\right|_{A} \rightarrow A$. It follows that $\mathbf{Z}_{2^{*}}$ । acts freely on det $\left.\nu\right|_{A} \rightarrow A$; hence, $\left.\operatorname{det} \nu\right|_{A} \rightarrow A$ bounds. Because $w\left(\left.\operatorname{det} \nu\right|_{A}\right)=1+u_{1}$, $u_{1}^{i} w_{\omega}(A)[A]=0$. In particular, $0=\left\{w(A)\left(1+u_{1}\right)\right\}_{\omega}[A]=w_{\omega}(B)[B]$ since according to Stong [5], $\mathbf{R} P(\nu) \rightarrow \mathbf{R} P^{\infty}$ bounds.

$$
\begin{aligned}
w(\mathbf{R} P(\nu+1)) & =w(F)\left\{(1+c)^{3}+u_{1}(1+c)^{2}+u_{2}(1+c)\right\} \\
& =w(F)\left\{1+\left(c+u_{1}\right)+\left(c^{2}+u_{2}\right)\right\},
\end{aligned}
$$

since $c^{3}+u_{1} c^{2}+u_{2} c=0$. Recall that $[\mathbf{R} P(\nu+1)]=[M]$ in $\mathfrak{R}_{*}$.

$$
\begin{gathered}
w_{n}[\mathbf{R} P(\nu+1)]=w_{n-2}(F)\left(c^{2}+u_{2}\right)[\mathbf{R} P(\nu+1)]=w_{n-2}(F)[F]=0, \\
w_{1} w_{n-1}[\mathbf{R} P(\nu+1)]=\left(w_{1}(F)+c+u_{1}\right)\left(w_{n-2}(F)\left(c+u_{1}\right)\right. \\
\left.+w_{n-3}(F)\left(c^{2}+u_{2}\right)\right)[\mathbf{R} P(\nu+1)] \\
=\left(2 u_{1} w_{n-3}(F)+w_{n-2}(F)+w_{n-3}(F) w_{1}(F)\right)[F]=0 .
\end{gathered}
$$

We know that $w_{1}^{n}[M]=0$, so assume $2 \leqslant i \leqslant n-2$.

$$
\begin{aligned}
w_{1}^{i} w_{n-i}(\mathbf{R} P(\nu+1))=\left(w_{1}(F)+c+u_{1}\right)^{i}\left(w_{n-i}(F)+w_{n-i-1}(F)\left(c+u_{1}\right)\right. & \left.+w_{n-i-2}(F)\left(c^{2}+u_{2}\right)\right) \\
= & \sum_{j=0}^{i}\left(\begin{array}{l}
i \\
j
\end{array}\right) c^{j}\left(w_{1}(F)+u_{1}\right)^{i-j}\left(w_{n-1}(F)+w_{n-i-1}(F) u_{1}+w_{n-i-2}(F) u_{2}\right) \\
& +\sum_{j=0}^{i}\left(\begin{array}{l}
i \\
j
\end{array}\right) c^{j}\left(w_{1}(F)+u_{1}\right)^{i-j}\left(w_{n-i-1}(F) c+w_{n-i-2}(F) c^{2}\right) .
\end{aligned}
$$

Now,

$$
\begin{gathered}
c^{j}\left(w_{1}(F)+u_{1}\right)^{i-j}\left(w_{n-i}(F)+w_{n-i-1}(F) u_{1}\right)[\mathbf{R} P(\nu+1)] \\
=\bar{u}_{j-2} w_{1}(\mathbf{R} P(\nu))^{i-j} w_{n-i}(\mathbf{R} P(\nu))[F]=0,
\end{gathered}
$$

where $\bar{u}=1 /\left(1+u_{1}+u_{2}\right)$. 


$$
\begin{aligned}
w_{1}^{i} w_{n-i}[\mathbf{R} & P(\nu+1)] \\
= & \sum_{j=2}^{i}\left(\begin{array}{l}
i \\
j
\end{array}\right) c^{j-1}\left(w_{1}(F)+u_{1}\right)^{i-j} w_{n-i-2}(F)\left(c^{3}+c^{2} u_{1}\right)[\mathbf{R} P(\nu+1)] \\
& +\sum_{j=0}^{i}\left(\begin{array}{l}
i \\
j
\end{array}\right) c^{j}\left(w_{1}(F)+u_{1}\right)^{i-j}\left(w_{n-i-1}(F) c+w_{n-i-2}(F) c^{2}\right)[\mathbf{R} P(\nu+1)] \\
= & \sum_{j=2}^{i}\left(\begin{array}{l}
i \\
j
\end{array}\right) c^{j+1}\left(w_{1}(F)+u_{1}\right)^{i-j}\left(w_{n-i-2}(F) u_{1}+w_{n-i-1}(F)\right)[\mathbf{R} P(\nu+1)] \\
& +\sum_{j=0}^{1}\left(\begin{array}{l}
i \\
j
\end{array}\right) c^{j}\left(w_{1}(F)+u_{1}\right)^{i-j}\left(w_{n-i-1}(F) c+w_{n-i-2}(F) c^{2}\right)[\mathbf{R} P(\nu+1)] \\
= & \left\{\left(w_{1}(F)+u_{1}\right)^{i} w_{n-i-2}(F)+i\left(w_{1}(F)+u_{1}\right)^{i-1} w_{n-i-1}(\mathbf{R} P(\nu))\right\}[F] \\
= & \left(w_{1}(A)+u_{1}\right)^{i} w_{n-i-2}(A)[A]+w_{1}(B)^{i} w_{n-i-2}(B)[B]=0 .
\end{aligned}
$$

Thus, $w_{1}^{i} w_{n-i}[M]=0$ for $0 \leqslant i \leqslant n$.

Set $J=\left\{\alpha: w_{1}^{i} w_{n-i}(\alpha)=0,0 \leqslant i \leqslant n\right\}$. According to Capobianco [3], $\alpha \in J$ can be represented by a fibering with fiber $\mathbf{R} P(3)$, and Stong [6] exhibits generators of $J$; they are of the form $\mathbf{R} P(\xi+\eta)$, where $\xi$ is a complex line bundle and $\eta$ is a real 2-plane bundle. There is a $\mathbf{Z}_{2}$, action on $\mathbf{R} P(\xi+\eta)$ induced by multiplication by the $2^{s}$ th roots of 1 in the fibers of $\xi$, and by 1 in the fibers of $\eta$. The fixed set is $\mathbf{R} P(\xi) \cup \mathbf{R} P(\eta)$, which has codimension 2 ; thus, $J=I_{2^{\prime}}$.

Proposition 2.4. If $S$ is a 2-group containing $\mathbf{Z}_{2} \times \mathbf{Z}_{2}$, then $I_{S}=0$ in $\mathfrak{R}_{*}$.

Proof. If $M \rightarrow M / S$ is a branched covering, then the isotropy groups of the $S$ action on $M$ are cyclic. In particular, $\mathbf{Z}_{2} \times \mathbf{Z}_{2}$ acts without stationary points, implying $[M]=0$ in $\mathfrak{N}_{*}[\mathbf{4}, 30.1]$.

REMARK. If $S$ is a nonabelian 2-group that does not contain $\mathbf{Z}_{2} \times \mathbf{Z}_{2}$, then $S$ is a generalized quaternion group.

Let $Q_{8}=\{ \pm 1, \pm i, \pm j, \pm i j\}$ be the quaternion group, let $M^{n} \rightarrow M^{n} / Q_{8}$ be a branched covering and let $F^{n-2}$ be the fixed point set. Then, $F=A_{2} \cup B_{i} \cup C_{j} \cup$ $D_{i j}$, where $A_{2}$ is the fixed point set of $\mathbf{Z}_{2}$ in $Q_{8}, B_{i}$ is the fixed point set of $\langle i\rangle$ in $Q_{8}$, etc.

$\langle j\rangle$ gives us a $\mathbf{Z}_{4}$ action on the normal bundle of $B_{i}, \nu \rightarrow B$, such that $\mathbf{Z}_{2} \subset \mathbf{Z}_{4}$ acts trivially on $B$ and as multiplication by -1 in the fibers of $\nu$. Also, $\mathbf{Z}_{4} / \mathbf{Z}_{2} \simeq \mathbf{Z}_{2}$ acts freely on $B$.

Beem [2] supplies us with a classifying space for such bundles: Write $\mathbf{C}^{\infty}=\mathbf{R}^{\infty}+$ $j \mathbf{R}^{\infty}$. Then $j$ acts on $\mathbf{C}^{\infty}$ by multiplication. There is an induced action on $\gamma^{2} \rightarrow$ $\mathrm{BO}_{2}\left(\mathbf{C}^{\infty}\right)$ such that $\mathbf{Z}_{2} \subset\langle j\rangle$ acts trivially on $B O\left(\mathbf{C}^{\infty}\right)$ and as multiplication by -1 in the fibers of $\gamma^{2}$. We can classify a free $\mathbf{Z}_{2}$ action by a map into [ $\left.S^{\infty},-1\right]$. Thus, the desired classifying space is $\mathrm{BO}_{2}\left(\mathbf{C}^{\infty}\right) \times S^{\infty} / \mathbf{Z}_{2}$. Beem [2] determines that

$$
H^{*}\left(B O_{2}\left(\mathbf{C}^{\infty}\right) \times S^{\infty} / \mathbf{Z}_{2} ; \mathbf{Z}_{2}\right)=\mathbf{Z}_{2}\left[w_{1}, w_{2}^{2}, \alpha\right] /\left\langle\alpha^{2} w_{1}\right\rangle
$$


where $w_{\mathrm{i}}, w_{2}$ are the universal classes which generate $\mathrm{H}^{*}\left(\mathrm{BO}_{2} ; \mathbf{Z}_{2}\right)$ and $\alpha$ generates $H^{*}\left(\mathbf{R} P^{\infty} ; \mathbf{Z}_{2}\right)$.

We have the following commutative diagram:

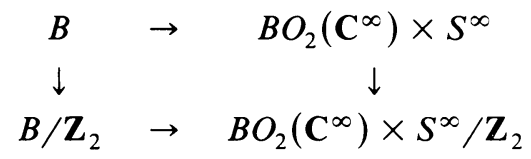

The classes $w_{1}$ and $w_{2}^{2}$ pull back to $u_{1}$ and $u_{2}^{2}$ in $H^{*}\left(B ; \mathbf{Z}_{2}\right)$, where $w(\nu)=1+u_{1}+$ $u_{2}$. Note that $u_{1}=0$ because $\nu \rightarrow B$ is a complex vector bundle. Thus, $w_{\omega} u_{2}^{2 j}[B]=0$ for all $\omega, j$ since these numbers come from $B / \mathbf{Z}_{2}$. We obtain the same result for $A, C$, and $D$.

In fact, for $A$ we can say more. There is a $Q_{8}$ action on $\gamma^{2} \rightarrow B O_{2}\left(\mathbf{H}^{\infty}\right)$ induced by multiplication such that $\mathbf{Z}_{2} \subset Q_{8}$ acts trivially on $\mathrm{BO}_{2}\left(\mathbf{H}^{\infty}\right)$ and as multiplication by -1 in the fibers of $\gamma^{2}$. We have such an action on $\nu \rightarrow A$; also, $Q_{8} / \mathbf{Z}_{2} \simeq \mathbf{Z}_{2} \times \mathbf{Z}_{2}$ acts freely on $A$. Such an action can be classified by a map into $\left[S^{\infty} \times S^{\infty} ;-1 \times 1,1\right.$ $\times-1]$. Thus, we have a classifying space for $A / \mathbf{Z}_{2} \times \mathbf{Z}_{2}$, namely $B O_{2}\left(\mathbf{H}^{\infty}\right) \times S^{\infty} \times$ $S^{\infty} / i \times-1 \times 1, j \times 1 \times-1$, and $H^{*}\left(B O_{2}\left(\mathbf{H}^{\infty}\right) \times S^{\infty} \times S^{\infty} / \mathbf{Z}_{2} \times \mathbf{Z}_{2} ; \mathbf{Z}_{2}\right) \simeq$ $\mathbf{Z}_{2}\left[\alpha, \beta, w_{1}, w_{2}^{4}\right] /$ relations.

From the double covers $A \rightarrow A / \mathbf{Z}_{2}$ and $\mathrm{BO}_{2}\left(\mathbf{C}^{\infty}\right) \times S^{\infty} \rightarrow \mathrm{BO}_{2}\left(\mathbf{C}^{\infty}\right) \times S^{\infty} / \mathbf{Z}_{2}$, we have the following exact sequences:

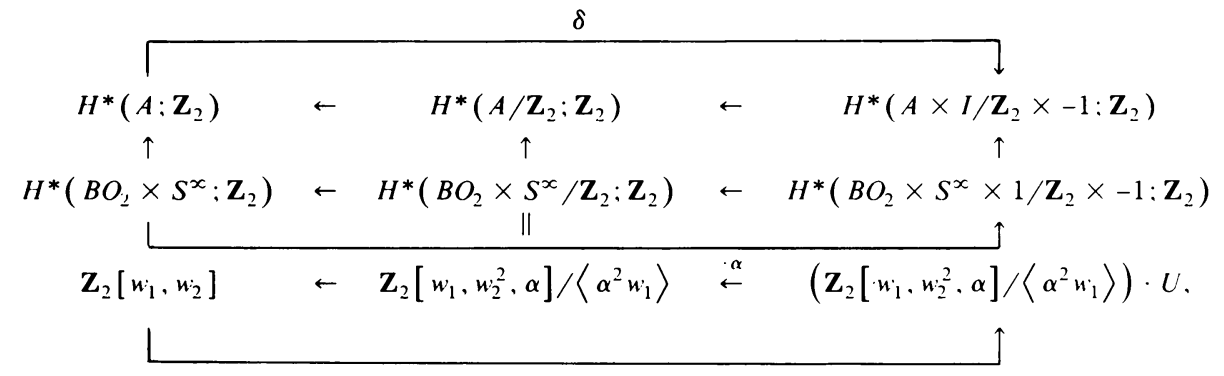

where $U$ is the Thom class of the line bundle $B O_{2}\left(\mathbf{C}^{\infty}\right) \times S^{\infty} \times 1 / \mathbf{Z}_{2} \times-1 \rightarrow$ $\mathrm{BO}_{2}\left(\mathbf{C}^{\infty}\right) \times S^{\infty} / \mathbf{Z}_{2}$.

$$
\begin{aligned}
\left\langle w_{\omega} u_{1}^{r} u_{2}^{4 j+1},[A]\right\rangle & =\left\langle w_{\omega} u_{1}^{r} u_{2}^{4 j+1}, \partial\left[A \times I / \mathbf{Z}_{2} \times-1\right]\right\rangle \\
& =\left\langle\delta\left(w_{\omega} u_{1}^{r} u_{2}^{4 j+1}\right),\left[A \times I, \mathbf{Z}_{2} \times-1\right]\right\rangle \\
& =\left\langle w_{\omega} u_{1}^{r} u_{2}^{4 j}\left(\delta u_{2}\right),\left[A \times I, \mathbf{Z}_{2} \times-1\right]\right\rangle .
\end{aligned}
$$

In the cohomology of the universal space, $\delta w_{2}$ is a class such that $\alpha\left(\delta w_{2}\right)=0$; thus, $\delta w_{2}=\alpha w_{1} U$, implying

$$
\begin{aligned}
\left\langle w_{\omega} u_{1}^{r} u_{2}^{4 j+1},[A]\right\rangle & =\left\langle w_{\omega} u_{1}^{r} u_{2}^{4 j} \alpha u_{1} U,\left[A \times I / \mathbf{Z}_{2} \times-1\right]\right\rangle \\
& =\left\langle w_{\omega} u_{1}^{r+1} u_{2}^{4 j} \alpha,\left[A / \mathbf{Z}_{2}\right]\right\rangle=0
\end{aligned}
$$

since $u_{2}^{4 j}$ comes from $H^{*}\left(A / \mathbf{Z}_{2} \times \mathbf{Z}_{2} ; \mathbf{Z}_{2}\right)$.

We consider equivariant bordism of our special $Q_{8}$ actions, that is, $Q_{8}$ actions such that each action is free off of a codimension 2 submanifold, and on that 
submanifold the isotropy groups are nontrivial cyclic groups. We obtain ConnerFloyd exact sequences:

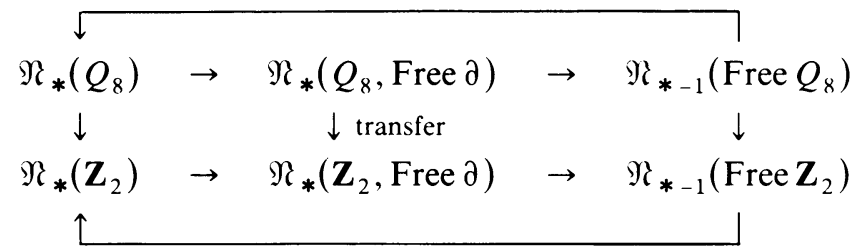

where the vertical arrows are obtained by restricting to the $\mathbf{Z}_{2} \subset Q_{8}$ action. Classification of the normal bundle to the fixed set leads to

$$
\begin{aligned}
\mathfrak{N}_{*}\left(Q_{8}, \text { Free } \partial\right) \simeq & \mathfrak{N}_{*-2}\left(B O_{2}\left(\mathbf{H}^{\infty}\right) \times S^{\infty} \times S^{\infty} / \mathbf{Z}_{2} \times \mathbf{Z}_{2}\right) \\
& +3 \mathfrak{N}_{*-2}\left(B U_{1}\left(\mathbf{H}^{\infty}\right) \times S^{\infty} / \mathbf{Z}_{2}\right),
\end{aligned}
$$

and $\mathfrak{N}_{*}\left(\mathbf{Z}_{2}\right.$, Free $\left.\partial\right) \simeq \mathfrak{N}_{*-2}\left(B O_{2}\left(\mathbf{H}^{\infty}\right)\right)$. A class in $\mathfrak{N}_{*-2}\left(B O_{2}\right)$ is determined by the numbers $w_{\omega} u_{1}^{r} u_{2}^{s}[F]$; in fact, the numbers $w_{\omega} u_{1}^{r} u_{2}^{s}[A]$ determine a class in the image of transfer: Since $\mathbf{R} P(\nu) \rightarrow \mathbf{R} P^{\infty}$ bounds,

$$
0=c^{4 j+3} w_{\omega}[\mathbf{R} P(\nu)]=\bar{u}_{4 j+2}\left\{\left(1+u_{1}\right) w(A)\right\}_{\omega}[A]+u_{2}^{2 j+1} w_{\omega}[B \cup C \cup D] .
$$

\section{LEMMA 2.5. The image of the transfer map}

$$
\operatorname{tr}: \mathfrak{N}_{*}\left(B \mathrm{O}_{2}\left(\mathbf{H}^{\infty}\right) \times S^{\infty} \times S^{\infty} / \mathbf{Z}_{2} \times \mathbf{Z}_{2}\right) \rightarrow \mathfrak{N}_{*}\left(B O_{2}\left(\mathbf{H}^{\infty}\right)\right)
$$

is a free $\mathfrak{N}_{*}$-module with a basis in the image of the homology transfer.

Proof. Let $\pi: \tilde{X} \rightarrow X$ be a double cover. There is a line bundle $l$ associated to this cover with $E l=\tilde{X} \times \mathbf{R} / \mathbf{Z}_{2} \times-1$. Let $[M, f] \in \mathfrak{N}_{*}(X)$; then, we have a double cover of $M, \tilde{M}=\{(m, \tilde{x}) \in M \times \tilde{X}: f(m)=\pi(\tilde{x})\}$. The Thom isomorphism $T$ : $\mathfrak{R}_{*}(X)=\tilde{\mathfrak{R}}_{*+1}(T l) \simeq \mathfrak{N}_{*+1}(D l, S l)$ sends $[M]$ to $\left[\tilde{M} \times I / \mathbf{Z}_{2} \times-1, \tilde{M} \times\right.$ $\left.\{ \pm 1\} / \mathbf{Z}_{2} \times-1\right]$, and the boundary homomorphism $\partial: \mathfrak{N}_{*+1}(D l, S l) \rightarrow \mathfrak{N}_{*}(S l) \simeq$ $\mathfrak{N}_{*}(\tilde{X})$ sends the given pair to $[\tilde{M}]$. The composite $\partial T: \mathfrak{N}_{*}(X) \rightarrow \mathfrak{N}_{*}(\tilde{X})$ is just the transfer map associated to the double cover $\tilde{X} \rightarrow X$.

If $f: Y \rightarrow Z$ is a map, then the image of $f_{*}: \mathfrak{N}_{*}(Y) \rightarrow \mathfrak{N}_{*}(Z)$ is a free $\mathfrak{N}_{*}$-module with a basis in the image of $f_{*}: H_{*}(Y) \rightarrow H_{*}(Z)$. Now $\partial$ is the composite of $i_{*}$ : $\mathfrak{R}_{*+1}(D l, S l) \rightarrow \tilde{\mathfrak{N}}_{*+1}(\Sigma S l)$, where $i$ is the inclusion, and the suspension isomorphism $\sigma: \tilde{\mathfrak{N}}_{*+1}(\Sigma S l) \rightarrow \mathfrak{N}_{*}(S l)$. Therefore, the image of $\partial T: \mathfrak{N}_{*}(X) \rightarrow \mathfrak{N}_{*}(\tilde{X})$ is a free $\mathfrak{R}_{*}$-module with a basis in the image of the homology transfer. It follows that this result holds for the transfer map associated to the double-double cover, $\mathrm{BO}_{2}\left(\mathbf{H}^{\infty}\right) \times S^{\infty} \times S^{\infty} \rightarrow \mathrm{BO}_{2}\left(\mathbf{H}^{\infty}\right) \times S^{\infty} \times S^{\infty} / \mathbf{Z}_{2} \times \mathbf{Z}_{2}$.

We now need to find a basis for the image of the homology transfer. Consider the fibering $\mathbf{R} P^{3} \rightarrow \mathbf{R} P\left(\gamma_{2 j+1}^{\mathrm{cx} 1} \oplus \gamma_{n}^{1} \oplus \gamma_{m}^{1}\right) \rightarrow \mathbf{C} P^{2 j+1} \times \mathbf{R} P^{n} \times \mathbf{R} P^{m} . N S^{1}$ in $S^{3}$ acts on $\gamma_{2 j+1}^{\mathrm{cx} 1} \rightarrow \mathbf{C} P^{2 j+1}$ by multiplication, and it acts on $\gamma_{n}^{1}+\gamma_{m}^{1} \rightarrow \mathbf{R} P^{n} \times \mathbf{R} P^{m}$ trivially. In the induced action on $\mathbf{R} P\left(\gamma_{2 j+1}+\gamma_{n}+\gamma_{m}\right), S^{1}$ fixes $\mathbf{R} P\left(\gamma_{n}+\gamma_{m}\right)$, and $\mathbf{Z}_{2} \subset S^{1}$ fixes $\mathbf{R} P\left(\gamma_{2 j+1}\right) \simeq \mathbf{R} P^{4 j+3} \times \mathbf{R} P^{n} \times \mathbf{R} P^{m}$, both codimension 2 submanifolds. Thus, we have a $Q_{8} \subset N S^{1}$ action on $\mathbf{R} P\left(\gamma_{2 j+1}+\gamma_{n}+\gamma_{m}\right)$ that induces a branched covering. The normal bundle of $\mathbf{R} P\left(\gamma_{2 j+1}\right)$ in $\mathbf{R} P\left(\gamma_{2 j+1}+\gamma_{n}+\gamma_{m}\right)$ is 
$\nu=\mu \otimes\left(\gamma_{n} \oplus \gamma_{m}\right)$, where $\mu \rightarrow \mathbf{R} P^{4 j+3} \times \mathbf{R} P^{n} \times \mathbf{R} P^{m}$ is the pullback of the standard line bundle over $\mathbf{R} P^{4 j+3}$. Then,

$$
w(\nu)=(1+c+a)(1+c+b)=1+(a+b)+\left(c^{2}+c(a+b)+a b\right),
$$

with $a \in H^{1}\left(\mathbf{R} P^{n} ; \mathbf{Z}_{2}\right), b \in H^{1}\left(\mathbf{R} P^{m} ; \mathbf{Z}_{2}\right)$, and $c \in H^{1}\left(\mathbf{R} P^{4 j+3} ; \mathbf{Z}_{2}\right)$, the nonzero classes.

Let $n=0$; then, $w(\nu)=1+b+c(c+b)$, and $u_{1}^{r} u_{2}^{4 j+3}=b^{r}\left[c^{4 j+3}(c+b)^{4 j+3}\right]$ $=c^{4 j+3} b^{4 j+3+r}$. If $m \geqslant 4 j+3$,

$$
u_{1}^{m-(4 j+3)} u_{2}^{4 j+3}\left[\mathbf{R} P\left(\gamma_{2 j+1}\right)\right]=c^{4 j+3} b^{m}\left[\mathbf{R} P^{4 j+3} \times \mathbf{R} P^{m}\right]=1 .
$$

Since these numbers are the only possible nonzero numbers of the form $u_{1}^{r} u_{2}^{s}[A]$, we get all possible nonzero numbers $u_{1}^{r} u_{2}^{s}[A]$ by taking

$$
[M]=[\mathbf{R} P(0, m, 2 j+1,2 j+1)] \text {, with } m \geqslant 4 j+3 \text {. }
$$

Because $H^{*}\left(\mathrm{BO}_{2} ; \mathbf{Z}_{2}\right)=\mathbf{Z}_{2}\left[w_{1}, w_{2}\right]$, it follows that the homology classes corresponding to the $\left[\mathbf{R} P\left(\gamma_{2 j+1}\right), \nu\right]$ form a basis for the image of

$$
\operatorname{tr}: H_{*}\left(B O_{2}\left(\mathbf{H}^{\infty}\right) \times S^{\infty} \times S^{\infty} / \mathbf{Z}_{2} \times \mathbf{Z}_{2} ; \mathbf{Z}_{2}\right) \rightarrow H_{*}\left(B O_{2}\left(\mathbf{H}^{\infty}\right) ; \mathbf{Z}_{2}\right) \text {. }
$$

Proposition 2.6. $I_{Q_{8}}$ in $\mathfrak{N}_{*}$ is the ideal I generated by $[\mathbf{R} P(0, m, 2 j+1,2 j+1)]$, with $m \geqslant 4 j+3$.

Proof. The ideal $I$ is certainly contained in $I_{Q}$. If $[M] \in I_{Q}$, then $\left[M, \mathbf{Z}_{2}\right] \in$ $\mathfrak{N}_{*}\left(\mathbf{Z}_{2}\right)$ is determined by $[F, \nu] \in \mathfrak{N}_{*-2}\left(B O_{2}\right)$, which in turn is determined by $[A, \nu] \in \mathfrak{N}_{*-2}\left(\mathrm{BO}_{2}\right)$. By Lemma 2.5 , the collection of all such $[A, \nu]$ is a free $\mathfrak{N}_{*}$-module on a basis given by the homology images of $\nu: \operatorname{R} P\left(\gamma_{2 j+1}\right) \subset$ $\mathbf{R} P(0, m, 2 j+1,2 j+1), m \geqslant 4 j+3$. Thus, $I_{Q}=I$.

Since the above arguments can be used for branchings which come from actions by a generalized quaternion group, we have the following

CoRollaRY 2.7. $I_{Q_{2} r}, r \geqslant 3$, is the ideal in $\Re_{*}$ generated by

$$
[\mathbf{R} P(0, m, 2 j+1,2 j+1)], \quad m \geqslant 4 j+3 .
$$

REMARK. If $Q_{8}$ acts on $M^{n}$ so that $M \rightarrow M / Q$ is a branched covering, then $w_{1}^{s} w_{i} w_{j} w_{k}\left[M^{n}\right]=0$, where $s+i+j+k=n$. These numbers distinguish classes in $\mathfrak{N}_{*}$ through dimension 7 ; thus, $\left(I_{Q}\right)_{n}=0$ for $n \leqslant 7$. In fact, $\left(I_{Q}\right)_{8}=0$ because $\mathbf{R} P(0,3,1,1)$ bounds.

We find an indecomposable $[\mathbf{R} P(0,2 j+1,2 j+1, m)], m \geqslant 4 j+3$, in dimension $n \geqslant 9$ if $n$ is not of the form $2^{s}-3,2^{s}-2,2^{s}-1$, or $2^{s}$. Also, if $n$ is of this form, then $\left[\mathbf{R} P\left(2 j+1,2 j+1, n_{1}, n_{2}\right)\right], 4 j+5+n_{1}+n_{2}=n$, is decomposable.

\section{REFERENCES}

1. N. Brand, Necessary conditions for the existence of branched coverings, Invent. Math. 54 (1979), 1-10.

2. R. P. Beem, On the bordism of almost free $\mathbf{Z}_{2}$ k actions, Trans. Amer. Math. Soc. 225 (1977), 83-105.

3. F. L. Capobianco, Cobordism classes represented by fiberings with fiber $\mathbf{R} P(2 k+1)$, Michigan Math. J. 24 (1977), 185-192.

4. P. E. Conner and E. E. Floyd, Differentiable periodic maps, Springer-Verlag, Berlin, 1964.

5. R. E. Stong, Branched coverings I, Trans. Amer. Math. Soc. 276 (1983), 375-402.

6. __ On fibering of cobordism classes, Trans. Amer. Math. Soc. 178 (1973), 431-447.

Department of Mathematics, University of Virginia, Charlottesville, Virginia 22904 